\title{
Contribution à l'étude sédimentologique du cône- terrasse de la Prims, implications hydrodynamiques (Land de Sarre, Allemagne)
}

Avec la collaboration de Sylvain BRESSY, Charlotte CAMBRESY-BAESCH, Thibaud DIEHL, Bénédicte MICHEL, Cyrielle MULLER, Aude PERRIN

New evidences on the terrace staircase system of the River Sarre (Saar) between Sarrebourg (France, Lorraine) and the Mosel confluence at Konz (Germany, Rheinland-Pfalz)

Neuere Untergliederung des Terrassensystems der Saar zwischen Sarrebourg (Frankreich, Lothringen) und der Mündung in die Mosel bei Konz (Deutschland, Rheinland-Pfalz)

Pierre Serrat et Dominique Harmand

\section{(2) OpenEdition}

\section{Édition électronique}

URL : http://journals.openedition.org/rge/1538

DOI : $10.4000 /$ rge. 1538

ISSN : 2108-6478

Éditeur

Association des géographes de l'Est

Édition imprimée

Date de publication : 1 septembre 2007

ISSN : 0035-3213

Référence électronique

Pierre Serrat et Dominique Harmand, « Contribution à l'étude sédimentologique du cône-terrasse de la Prims, implications hydrodynamiques (Land de Sarre, Allemagne) », Revue Géographique de l'Est [En ligne], vol. 47 / 4 | 2007, mis en ligne le 16 janvier 2012, consulté le 08 septembre 2020. URL : http:// journals.openedition.org/rge/1538; DOI : https://doi.org/10.4000/rge.1538

Ce document a été généré automatiquement le 8 septembre 2020.

Tous droits réservés 


\section{Contribution à l'étude} sédimentologique du cône-terrasse de la Prims, implications hydrodynamiques (Land de Sarre, Allemagne)

Avec la collaboration de Sylvain BRESSY, Charlotte CAMBRESY-BAESCH, Thibaud DIEHL, Bénédicte MICHEL, Cyrielle MULLER, Aude PERRIN

New evidences on the terrace staircase system of the River Sarre (Saar) between Sarrebourg (France, Lorraine) and the Mosel confluence at Konz (Germany, Rheinland-Pfalz)

Neuere Untergliederung des Terrassensystems der Saar zwischen Sarrebourg (Frankreich, Lothringen) und der Mündung in die Mosel bei Konz (Deutschland, Rheinland-Pfalz)

Pierre Serrat et Dominique Harmand

\section{Cadre général de l'étude}

1 La Prims est un cours d'eau modeste de $55 \mathrm{~km}$ de longueur qui conflue avec la Sarre à proximité de la commune de Dillingen, non loin de la ville de Saarlouis (Allemagne). Son bassin versant, d'une superficie de seulement $1000 \mathrm{~km}^{2}$ (fig. 1), draine le versant méridional de l'Hunsrück (Negendank, 1978), puis le bassin permien de Sarre-Nahe (Saar-Nahe-Bergland), encore largement recouvert par les formations triasiques, et notamment par le Buntsandstein moyen et supérieur (Konzan et al., 1977). Le bassin versant de la Prims a la particularité de présenter de puissantes formations alluviales quaternaires; la nature, l'origine, et les conditions de mise en place de ces sédiments 
revêtent un grand intérêt pour la compréhension de ces paléoenvironnements pléistocènes.

Figure 1 : Localisation de la rivière Prims, affluent de la Sarre et de son bassin versant

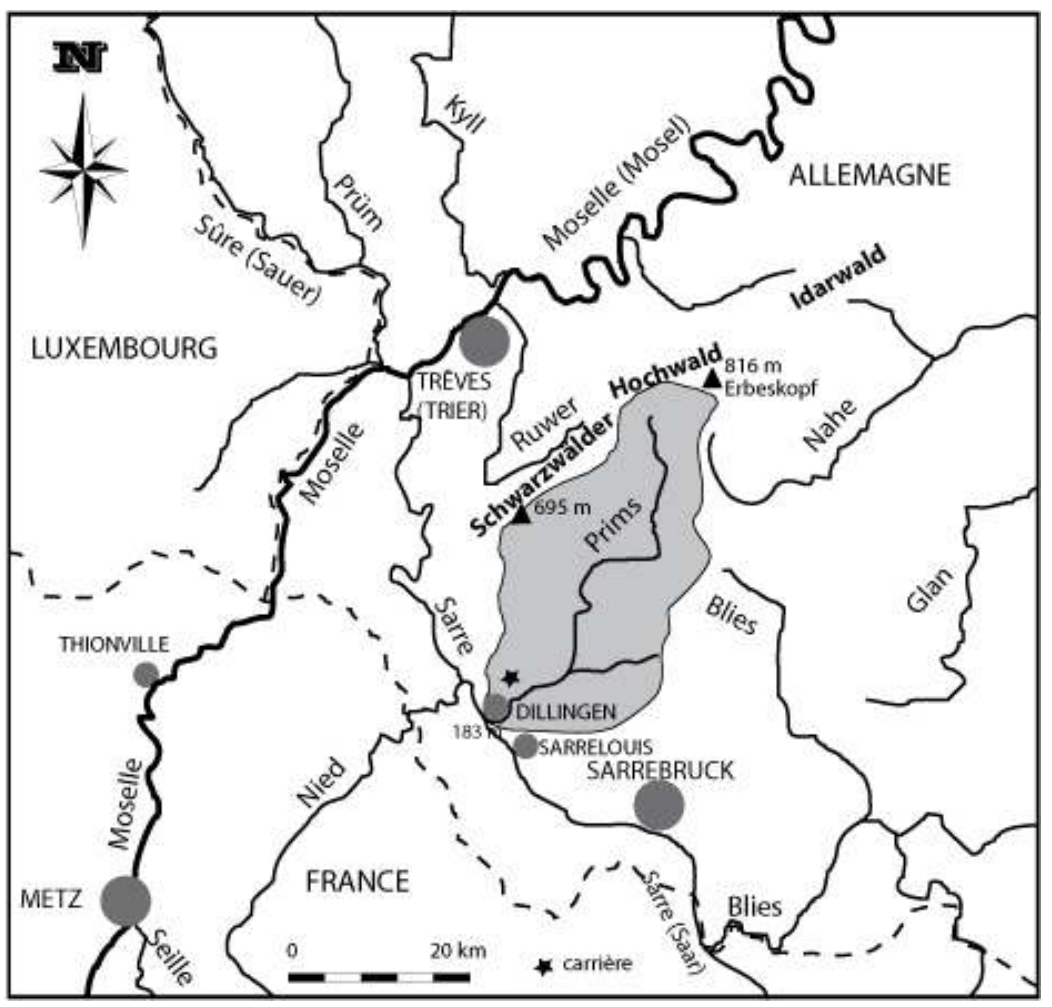

D’amont en aval, il est possible de distinguer trois parties dans la vallée de la Prims.

\section{A. Haute vallée de la Prims}

3 En amont, au sud de l'Hunsrück, sur la bordure nord du Saar-Nahe-Bergland, le bassin versant de la Prims est caractérisé par la présence de cônes de piedmont construits par les affluents de direction méridienne de la Prims. Ces cônes sont formés de formations alluviales grossières de 2 à $4 \mathrm{~m}$ de puissance désignées sous le terme de «Losheimer Schotterflur » (Zöller, 1985). Actuellement, la Prims et ses affluents coulent dans des vallées encaissées d'une vingtaine à une cinquantaine de mètres en contrebas des cônes situés en inversion de relief si bien que ces cônes appartiendraient à deux générations de terrasses, respectivement les terrasses moyennes et les terrasses principales (Fischer, 1957 ; Zöller, 1985). La conservation de ces cônes de piedmont est due à des facteurs morphostructuraux. Ils se trouvent, en effet, dans un compartiment affaissé situé le long de la faille de Metz-Mayence, dans une dépression orthoclinale modelée dans les grès tendres du Buntsandstein (Couches intermédiaires) et qui est fermée au sud et à l'est par la côte du Grès à Voltzia et par la pseudo-côte des mélaphyres du synclinal de la Prims (Liedtke, 1998). 


\section{B. Moyenne vallée}

4 Dans sa partie moyenne, la Prims coule dans une vallée étroite, de direction nord-sud. Cette vallée, bordée de quelques terrasses, est encaissée d'une centaine de mètres en contrebas des plateaux d'érosion du niveau de Lebach qui se situe à environ $300 \mathrm{~m}$ d'altitude absolue (Liedtke, 1998).

\section{Basse vallée}

Dans sa partie inférieure, la Prims coule dans une vallée plus large (1 km à Dillingen) en contrebas d'un dispositif de cônes terrasses étagés dans lesquels les auteurs ont distingué une basse terrasse, deux moyennes terrasses en rive gauche, et une terrasse principale en rive droite (Fischer, 1957 ; Konzan et al, 1977). A ce dispositif, Zöller (1985) ajoute une terrasse intermédiaire, entre la terrasse principale (notée « $M »$ ) et les moyennes terrasses (fig. 2). La terrasse principale « $M$ » s'étend sur une longueur d'environ $6 \mathrm{~km}$ et sur une largeur de $4 \mathrm{~km}$ et s'abaisse de $260 \mathrm{~m}$ à $230 \mathrm{~m}$. L'épaisseur des alluvions atteint $16 \mathrm{~m}$ à Diefflen (Zöller, 1985). L'extension de cette terrasse et la puissance des alluvions ont incité les auteurs allemands à rattacher le cône-terrasse de Diefflen aux terrasses principales de la Moselle. Pourtant ces dernières se localisent à des altitudes relatives plus élevées que le cône-terrasse de la Prims. Les altitudes relatives sont respectivement supérieures à $100 \mathrm{~m}$ dans le Massif schisteux et seulement de $+50 / 60 \mathrm{~m}$ non loin de la confluence Prims-Sarre. 
Figure 2 : Cartographie des formations alluviales pléistocènes (d'après Zöller, 1985) dans le bassin versant de la Prims et à proximité de la confluence avec la Sarre

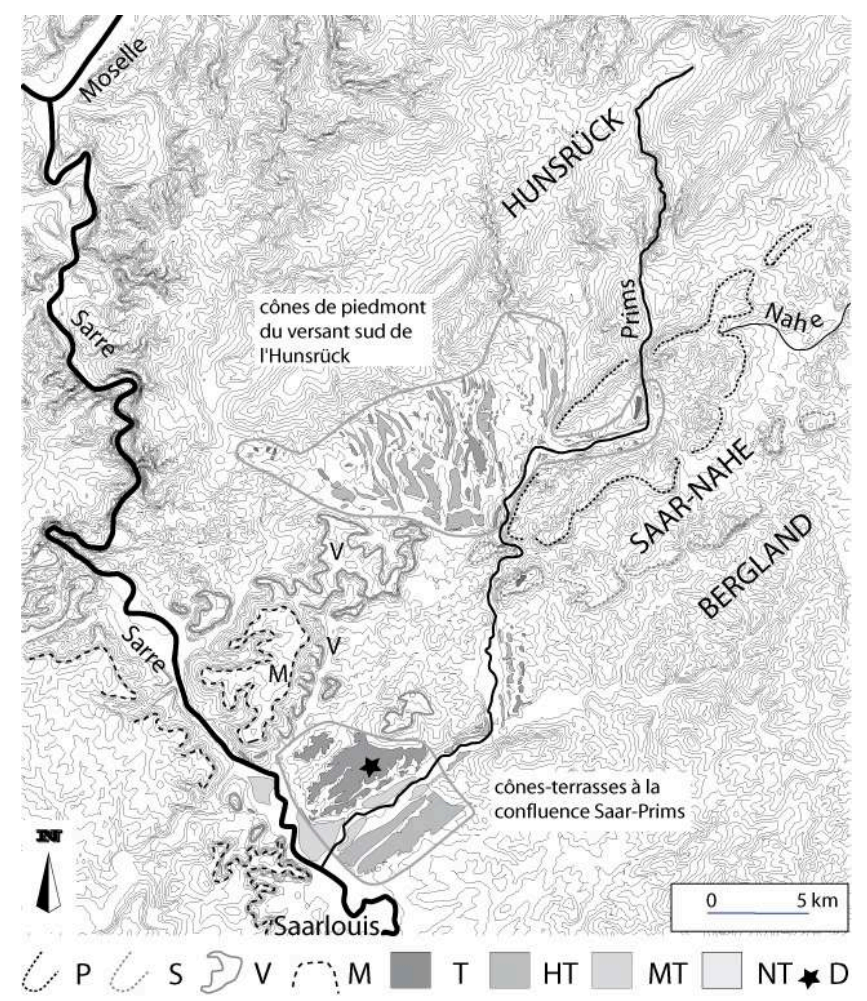

Reliefs du Saar-Nahe-Bergland :

$P$ : pseudo-côte des mélaphyres

$S$ : autres reliefs

Reliefs du bassin de Paris :

$\checkmark$ : côte du Grès à Voltzia

M : côte du Muschelkalk (d'après Liedtke, 1998)

Dispositif et âges présumés des formations alluviales :

$\mathrm{T}$ : Terrasses présumées d'âge tertiaire

$\mathrm{HT}$ : terrasses principales

MT : terrasses moyennes

NT : basses terrasses

$D$ : coupe de Diefflen

6 Les alluvions de la terrasse principale ont été étudiées par Zöller (1984). L'auteur a notamment distingué un sol interglaciaire dans la partie supérieure des alluvions et deux autres paléosols dans le loess qui recouvre ces dernières. Ces alluvions n'ont toutefois pas fait l'objet de nouvelles recherches depuis 25 ans. L'ouverture récente d'une gravière à Diefflen en 2006 a permis d'effectuer une nouvelle étude sédimentologique des alluvions et d'en tirer des informations sur l'hydrodynamique fluviale de ce cours d'eau, au cours du Pléistocène. 


\section{Observations et analyses}

\section{A. Lithofaciès et analyses granulométriques}

7 La coupe étudiée se situe à Diefflen, localité située à environ $5 \mathrm{~km}$ de la confluence Sarre-Prims, au nord-est de l'agglomération de Saarlouis (feuille 6606, R=2455250, $\mathrm{H}=5472000$ ) à environ $242 \mathrm{~m}$ d'altitude.

À Diefflen, les dépôts ont plus de $12 \mathrm{~m}$ de puissance. Ils sont constitués par deux ensembles :

-à la base, des alluvions parfois grossières, de 9,5 m d'épaisseur totale, organisées en trois bancs d'épaisseur plurimétrique, séparés par des bancs sableux d'épaisseur réduite, inférieure à un mètre ;

- au sommet, une couverture loessique de $3 \mathrm{~m}$ d'épaisseur.

Le dépôt repose sur les grès peu consolidés du Buntsandstein, qui se présentent en lits de couleur beige, ocre à jaune clair.

10 La coupe a permis d'observer et de définir 6 unités sédimentaires (fig. 3). Plus de 50 échantillons ont été prélevés et une étude granulométrique des sables et des galets a été réalisée. Les six analyses retenues dans ce texte sont représentatives des unités A, C, $\mathrm{E}$ et $\mathrm{F}$.

Figure 3 : Coupe litho-stratigraphique de la terrasse principale de Diefflen : 6 unités (A à F) ont été mises en évidence. Les nombres cerclés correspondent aux analyses présentées (figure 4 et suivantes)

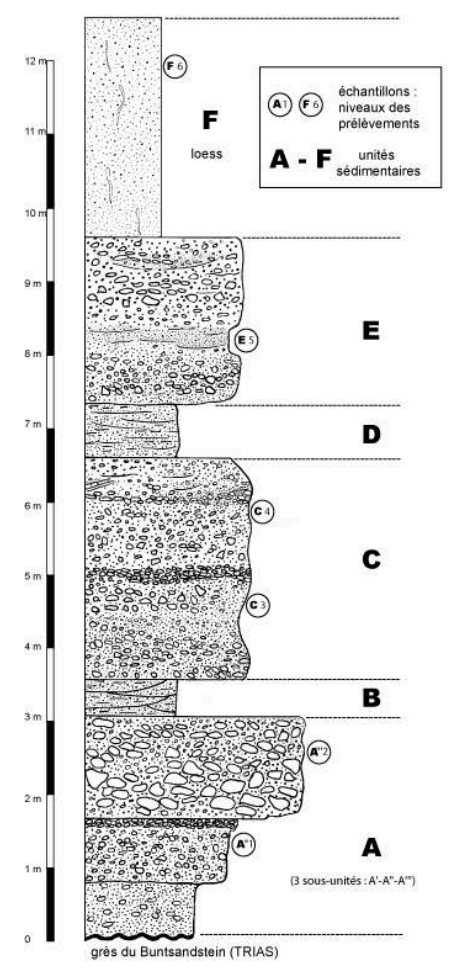


Figure 4 : Analyses granulométriques des sables dans l'unité $A$ (niveaux de prélèvements $A$ "1 et $A^{\prime \prime \prime 2}$ indiqués sur le log de la figure 3 )
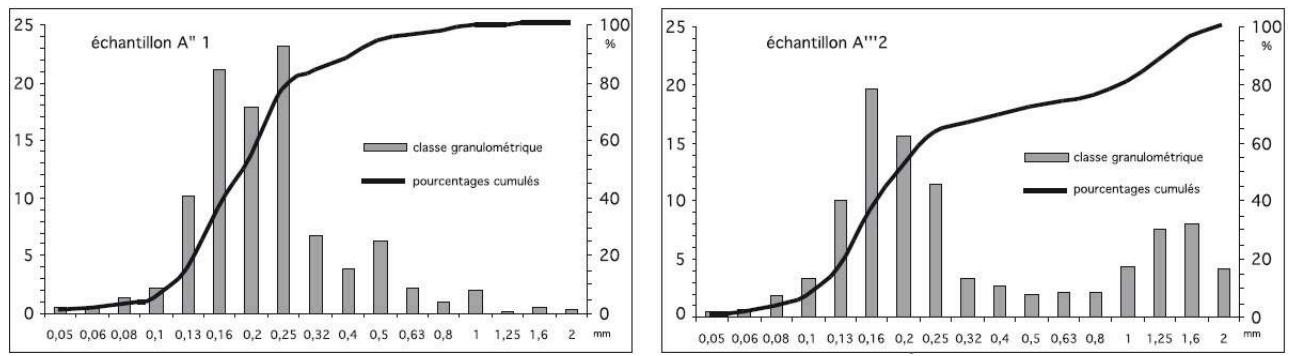

Figure 5 : Analyses granulométriques dans l'unité $C$ (niveaux de prélèvements $C 3$ et $C 4$ indiqués sur le log de la figure 3)
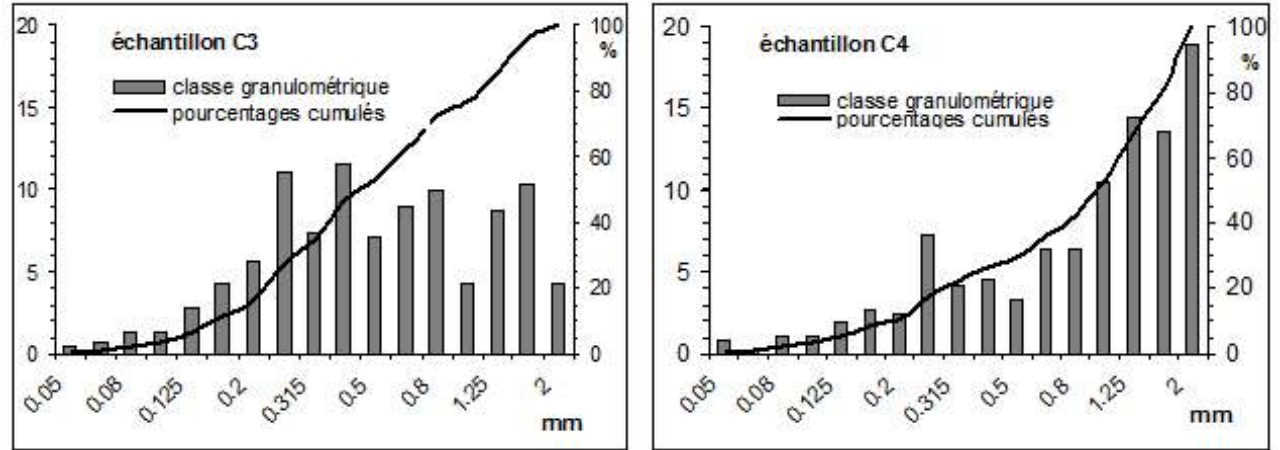
Les galets sont, quant à eux, majoritairement composés de quartz et quartzites issus du
socle dévonien (voir ci-dessous). Une grande majorité des galets de quartz présente des
formes anguleuses à sub-anguleuses. Ces galets n'ont donc pas subi une usure et une
abrasion très fortes, ce qui témoigne d'une origine assez proximale.
L'unité B est essentiellement constituée, sur une cinquantaine de centimètres

Au-dessus, on trouve une seconde sous-unité (désignée par A") composée d'un banc grossier contenant de nombreux galets arrondis à sub-arrondis, enrobés dans une matrice sablo-graveleuse (médiane des sables : $191 \mu \mathrm{m}$ ) (fig. 4, échantillon A"1). Un lit de graviers à texture ouverte d'une dizaine de cm d'épaisseur coiffe cette sous-unité.

La troisième sous-unité $\left(\mathrm{A}^{\prime \prime}\right)$ correspond à un banc très grossier, contenant des galets volumineux dont le grand axe peut dépasser $20 \mathrm{~cm}$. La taille moyenne de ces galets (le comptage a été effectué sur 103 galets) est de $64,2 \mathrm{~mm}$ pour le grand axe, de $45 \mathrm{~mm}$ pour la largeur et de $26,4 \mathrm{~mm}$ pour l'épaisseur, l'indice d'émoussé étant de 0,4. Les sables sont, quant à eux, mal classés (fig. 4, échantillon A"'2), ainsi que le montrent l'histogramme et la courbe cumulative $(\mathrm{Ft}=6,69 \mathrm{x}-7,25$ avec D50 $=195 \mu \mathrm{m})$. Il est à noter que la part des sables grossiers est beaucoup plus élevée $(28,5 \%)$ que dans l'échantillon précédent (6\%).

L'unité inférieure (A) présente une épaisseur de 3 mètres environ et se décompose en trois sous-unités ( $\left.\mathrm{A}^{\prime}-\mathrm{A}^{\prime \prime}-\mathrm{A}^{\prime \prime \prime}\right)$ globalement granocroissantes.

A la base, au contact des grès triasiques du Buntsandstein, se trouve une sous-unité ( $A^{\prime}$ ) composée de sables grossiers, de graviers auxquels s'ajoutent quelques galets. L'analyse de la matrice sableuse révèle une absence totale de classement granulométrique. d'épaisseur, de sables fins à moyens disposés généralement en lits subhorizontaux. Une 
datation des sables de cette unité par Résonance Paramagnétique Électronique (RPE), effectuée par le laboratoire du Museum national d'Histoire naturelle, est en cours.

17 L'unité C présente, sur 3 mètres d'épaisseur, des lithofaciès grossiers dans lesquels on observe des variations significatives de granularité. On a pu observer, parfaitement discernables, deux lits de graviers et galets à texture ouverte, sans aucune matrice.

L'analyse granulométrique de deux échantillons, prélevés dans parties les plus sableuses, montre, soit une absence de classement (fig. 5, échantillon C3) avec une courbe des fréquences cumulées quasiment rectiligne $(\mathrm{D} 50=451 \mu \mathrm{m})$, soit une prédominance de la fraction grossière à très grossière des sables $(70,3 \%$ avec D50 $=942$ $\mu \mathrm{m})$ (fig. 5 échantillon C4).

19 L'unité D rappelle l'unité B par sa faible épaisseur -environ $50 \mathrm{~cm}$ - et ses caractéristiques sédimentologiques. Elle est en effet formée de dépôts fins, sables et silts, et renferme des figures de cryoturbations, avec de remarquables involutions (fig. 6)de taille pluridécimétrique ( 20 à $40 \mathrm{~cm}$ ). Dans cette unité sableuse, un prélèvement devrait également permettre une datation RPE.

20 L'unité $\mathbf{E}$ est formée par une alternance de bancs et de lits sablo-graveleux se succédant sur une épaisseur de 2,5 $\mathrm{m}$ à $3 \mathrm{~m}$. Les galets sont assez peu allongés et leur taille peut atteindre 7 à $8 \mathrm{~cm}$ de longueur. Une analyse granulométrique pratiquée sur un lit intermédiaire plus sableux montre l'absence de sables fins ou très fins : $89 \%$ des sables ont en effet une taille supérieure à $315 \mu \mathrm{m}$ (fig. 7, échantillon E5).

21 L'unité sommitale $\mathbf{F}$, surtout présente dans la partie sud de la carrière, où son épaisseur peut atteindre $3 \mathrm{~m}$, est constituée essentiellement de limons grossiers et de sables fins à très fins, à litage à peine perceptible voire absent (fig. 8, échantillon F6). La fraction argileuse ne représente que $8,1 \%$. On y observe des traces verticales blanchâtres, à caractère pâteux et meuble. 
Figure 6 : Formes de cryoturbation, caractérisées par des involutions, dans le niveau sableux de l'unité stratigraphique D

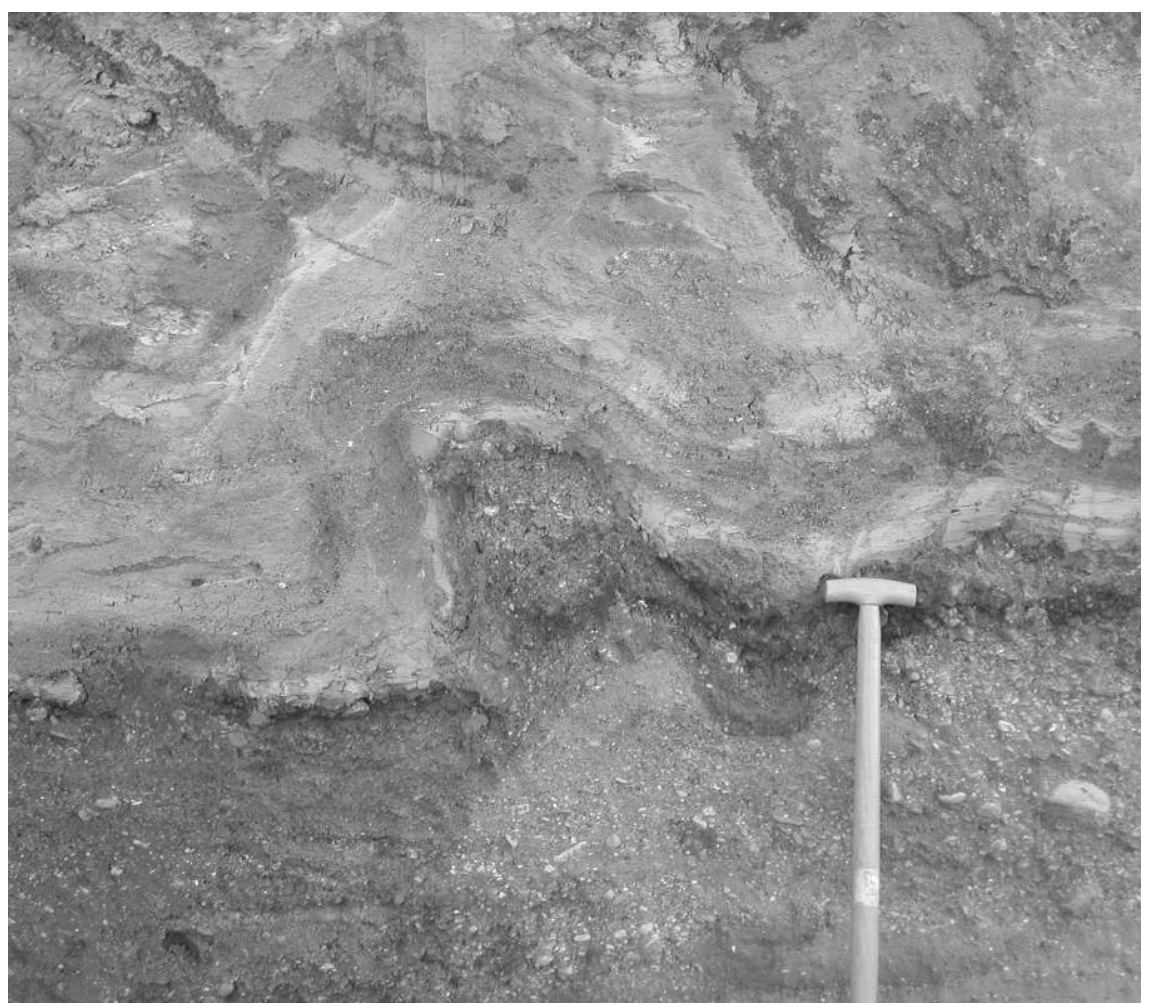

Figure 7 : Analyse granulométrique des sables dans l'unité $\mathrm{E}$ (niveau de prélèvement E5 indiqué sur le log de la figure 3)

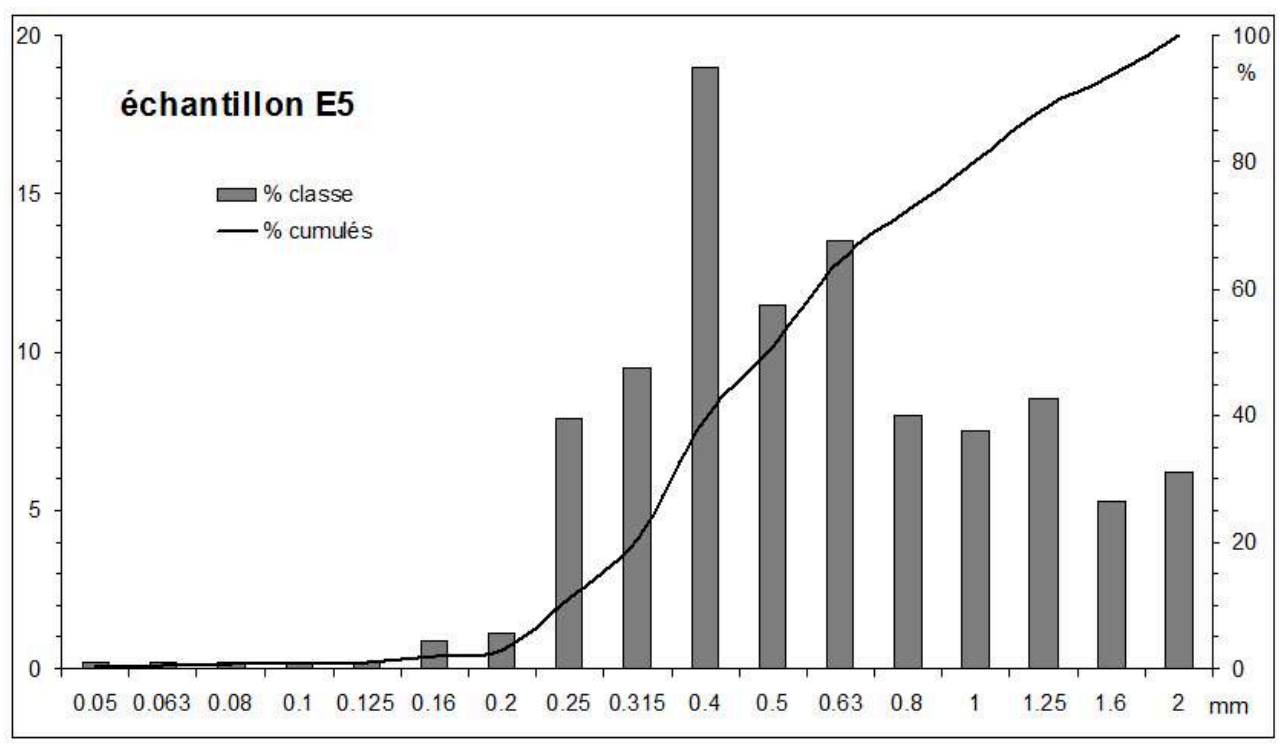


Figure 8 : Analyse granulométrique dans l'unité $\mathrm{F}$ (niveau de prélèvement $\mathrm{F} 6$ indiqué sur le log de la figure 3)

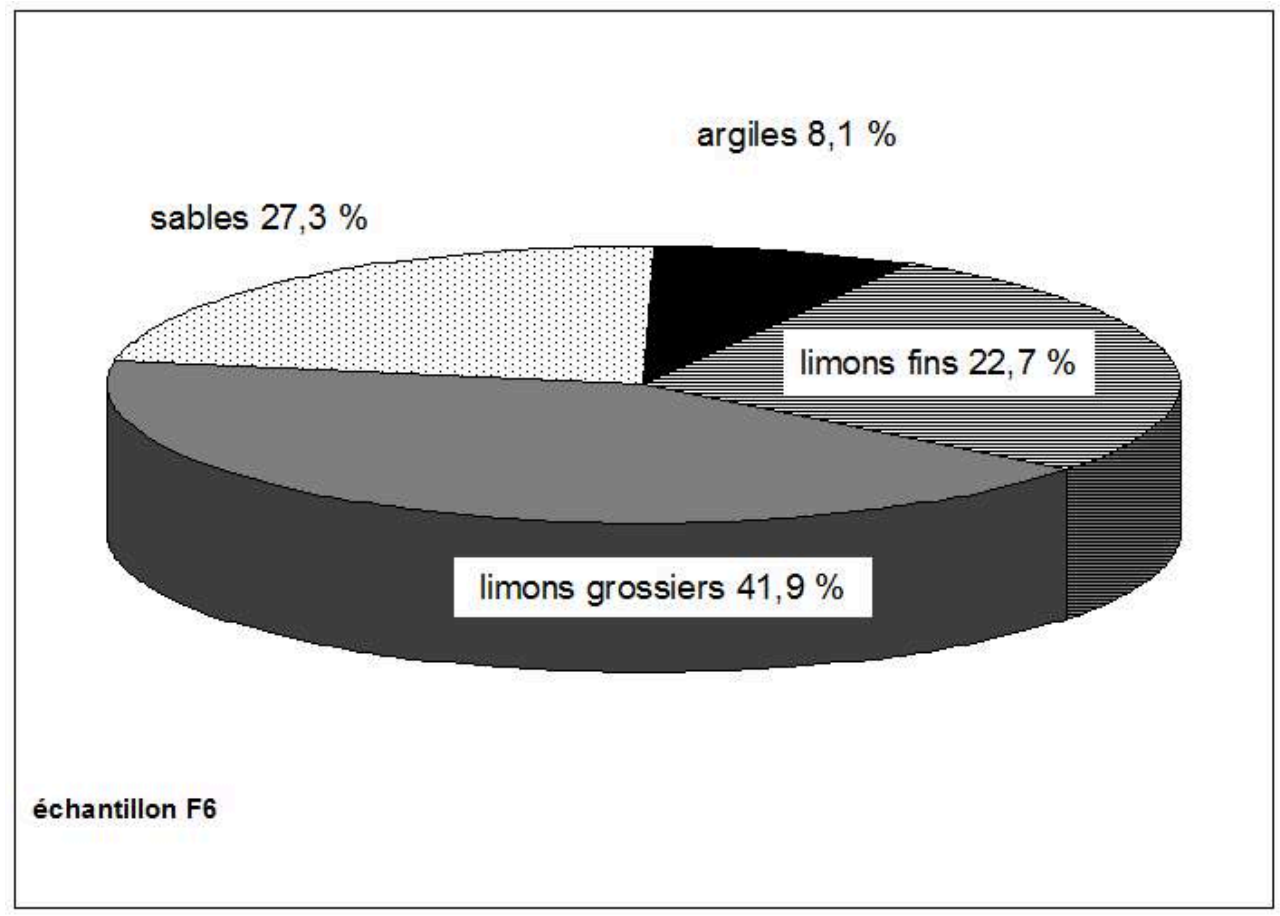

\section{B. Analyses calcimétriques et minéralogiques}

Des mesures calcimétriques effectuées sur les 5 unités de A à E, montrent que le carbonate de calcium est inexistant ou sous forme de traces ( $<1 \%)$, ce qui n'est guère surprenant puisque le bassin versant de la Prims est essentiellement inscrit dans des formations gréseuses et dans le socle hercynien de l'Hunsrück qui ne contient aucune formation carbonatée. En revanche, pour cette unité F, des valeurs de $\mathrm{CaCO} 3$ de l'ordre de $5 \%$ ont été observées. Cette analyse tendrait donc à prouver que ces éléments carbonatés ont une origine exogène: les apports les plus probables seraient donc éoliens. Il pourrait, en fait, s'agir de poussières relativement proximales, constituées surtout de limons, apportées par les vents d'ouest dominants. Ces limons carbonatés proviendraient des formations calcaires du Bassin parisien, en particulier du Muschelkalk qui affleure sur la bordure ouest du bassin versant de la Prims. En tout état de cause, cette unité $F$, très différente des unités sous-jacentes, présente effectivement toutes les caractéristiques d'un loess.

Dans l'unité $\mathrm{F}$, la minéralogie de la fraction inférieure à $50 \mu \mathrm{m}$, obtenue par diffractométrie aux rayons $\mathrm{X}$ (effectuée à l'Université de Perpignan), à partir d'un échantillon prélevé à environ $1 \mathrm{~m}$ de profondeur, révèle la prédominance du quartz (fig. 9) mais aussi la présence discrète de carbonates. Les minéraux argileux comme l'illite, la smectite ou la kaolinite sont très peu représentés. 
Figure 9 : Diffractométrie aux rayons $X$ de l'échantillon F6 dans l'unité $F$ (loess)

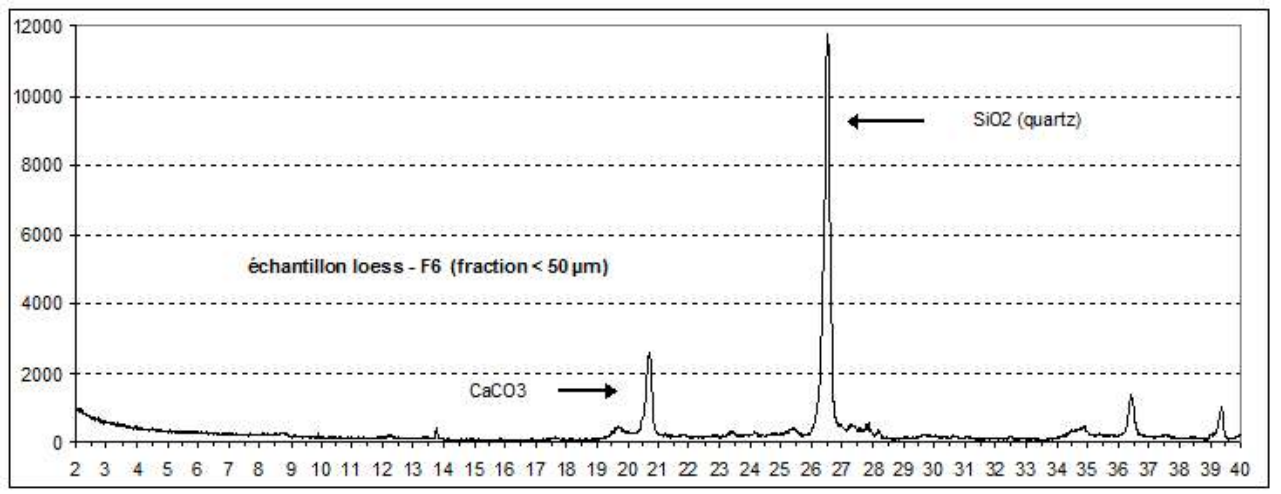

Les analyses minéralogiques effectuées à partir de quatre échantillons prélevés dans les sables des unités A', B, D et dans un banc sableux de l'unité E (Beiner et al., 2009), ont montré la prédominance de la tourmaline ( 47 à $56 \%$ ) et du zircon (24 à $38 \%$ ). Les autres minéraux sont constitués par les oxydes de titane (entre 6 et $13 \%$ ), le groupement métamorphique (staurolite, grenat, épidote : 1 à $5 \%$ ) et l'amphibole verte (1 à $4 \%$ ). La prédominance des ubiquistes confirme les analyses effectuées par les auteurs allemands à Diefflen (Zöller, 1985) et sur les terrasses principales de la vallée de la Sarre (Fischer, 1957). Ces résultats contrastent avec ceux obtenus par ces mêmes auteurs sur les terrasses plus basses plus riches en pyroxènes (entre 9 et $71 \%$ ).

Les minéraux lourds de la coupe de Diefflen sont issus du socle dévonien de l'Hunsrück, des formations permiennes du Saar-Nahe-Bergland, ainsi que des grès du Buntsandstein. L'absence des minéraux issus des vulcanites permiennes du bassin de Sarre-Nahe ("Saar-Nahe-Augit », Zöller, 1985) et qui existent dans les formations plus récentes est à mettre au compte d'une altération plus prononcée des alluvions de Diefflen.

Cette conclusion est conforme aux comptages lithologiques réalisés par Zöller (1985) sur des galets prélevés vers $11 \mathrm{~m}$ et $16 \mathrm{~m}$ de profondeur à proximité du terrain d'aviation de Diefflen (fraction 6,3-20 mm). En effet, le spectre pétrographique est caractérisé par l'abondance du quartz (à $11 \mathrm{~m}$ de profondeur : 54,3\% et à $16 \mathrm{~m}: 46,7 \%$ ) et du quartzite (respectivement $32 \%$ et $29,6 \%$ ), mais également par une proportion notable de roches volcaniques: "Porphyr" (respectivement 7,2 \% et 8,2 \%) et «Andesit » (respectivement 4,8 \% et 8,2 \%).

\section{Interprétations}

\section{A. Paléodynamiques fluviales : des écoulements de haute énergie}

La formation de Diefflen est constituée essentiellement d'unités grossières (A / C / E) dont le gradient granulométrique est parfois croissant (A). La dimension généralement élevée des galets montre que la Prims avait une compétence élevée.

De même, la très forte hétérométrie de la plupart des échantillons sableux étudiés (par exemple indice $\mathrm{Hq}=7,7$ pour l'échantillon $\mathrm{C} 4$ avec indice $\mathrm{Hq}=[\mathrm{Q} 3-\mathrm{Q} 1] / 2$ ) montre que la sédimentation s'est effectuée rapidement en raison de la densité et de la viscosité élevée des flux liquides et solides. 

régime immodéré marqué par des débits et une énergie élevés pendant des laps de temps probablement très courts. Le transport de matériaux grossiers par charriage de fond et la sédimentation se sont effectués lors de la débâcle estivale, en régime nival, dans un paléoenvironnement périglaciaire. Le caractère fluvio-glaciaire du dépôt pourrait cependant être envisagé en raison des formes caractéristiques de certains galets dans l'unité de base A (de type "fer à repasser ", ce qui atteste d'une origine proximale), de l'altitude élevée de l'Hunsrück (jusqu'à $800 \mathrm{~m}$ ) et de la proximité relative de l'inlandsis nord-européen (moins de $200 \mathrm{~km}$ pendant les glaciations du Saalien et de l'Elstérien) lors de la sédimentation. Toutefois, aucune forme et aucune formation glaciaires n'ont été mises en évidence sur l'Hunsrück.

En outre, les paléodynamiques fluviales expliquent l'extension et la puissance des alluvions du cône-terrasse de Diefflen et des cônes-terrasses postérieurs. Comme la Sarre s'écoule du SE vers le NW, perpendiculairement à son affluent la Prims qui coule du NE au SW, le débit plus élevé de la Sarre a fait obstacle au transfert des flux liquides et solides de la Prims, laquelle a déposé ses alluvions juste en amont de la confluence.

31 Toutefois, à cette cause hydrodynamique, s'ajoute le rôle joué par le cadre morphostructural : à la confluence Sarre-Prims, l'étalement des alluvions est favorisé par la présence en rive gauche de la Sarre des côtes du Grès à Voltzia et des calcaires du Muschelkalk que la Sarre traverse en percée cataclinale quelques kilomètres plus au nord, ce qui provoque un resserrement de la vallée.

\section{B. Tentatives de datations}

formation alluviale du cône-terrasse de la Prims témoigne de l'importance de la dynamique fluviatile en contexte périglaciaire au sud du Massif schisteux. Toutefois, il est impossible de situer ces phases d'aggradation dans le cycle glaciaire/interglaciaire.

Plusieurs arguments plaident en faveur d'un âge ancien, antérieur au Saalien, pour la formation alluviale de Diefflen. D'une part, l'altitude relative $(+60 \mathrm{~m})$ semble raccorder la terrasse de Diefflen aux terrasses des vallées de la Sarre supérieure et de la Moselle moyenne situées à la même altitude relative (Cordier et al., 2006; Harmand, 2007, ce volume). Le cône-terrasse de Diefflen a été rattaché à la terrasse $\mathrm{S} 6$ de la Sarre et à la terrasse M6 de la Moselle par ce dernier auteur. D'autre part, la formation de Diefflen, pauvre en pyroxène est antérieure à la rupture minéralogique qui sépare les alluvions des basses et moyennes terrasses riches en "Saar-Nahe-Augit» des alluvions plus élevées qui en sont pratiquement dépourvues, parce que plus altérées. Ensuite, les trois paléosols identifiés par Zöller (1984) au sommet des alluvions grossières (le plus ancien) et dans les loess (les deux plus récents) ont été raccordés à des interglaciaires antérieurs à l'Holsteinien, tandis que les déterminations paléomagnétiques réalisées sur des lits fins de Diefflen indiquent une polarité positive, ce qui milite en faveur d'un âge plus récent que l'inversion paléomagnétique Matuyama-Brunhes (environ 700000 ans).

Les alluvions de Diefflen, qui selon Zöller (1984) sont antérieures à 400000 ans, pourraient ainsi appartenir à l'Elstérien. Le même âge est attribué à la terrasse S6 de la Sarre par Harmand (2007, ce volume) qui rattacherait les alluvions de Diefflen au stade isotopique marin 12, en attendant les datations absolues RPE attendues sur les échantillons prélevés à Diefflen. 


\section{Conclusion}

L'épaisseur du dépôt de Diefflen, supérieure à 10 mètres, est tout à fait remarquable pour un bassin versant dont la superficie est d'un millier de $\mathrm{km}^{2}$ seulement. La puissance des alluvions et l'extension du cône-terrasse sont à attribuer à des conditions paléoenvironnementales périglaciaires lors d'une période froide bien marquée (stade isotopique 12 ?) et au cadre morphostructural marqué par des plateaux élevés dépassant $700 \mathrm{~m}$ d'altitude dans le haut bassin de la Prims. La remarquable préservation de ces formations apparait d'autant plus étonnante que le substratum est constitué de roches peu résistantes à l'érosion: les Couches intermédiaires sont effectivement mises en creux en avant de la côte du Grès à Voltzia dans le bassin de la Prims. Pourtant, il est relativement fréquent que des formations alluviales épaisses soient conservées sur des terrasses remarquablement planes, qu'il s'agisse de terrasses moyennes dans l'Est du bassin de Paris, ou de terrasses principales dans les vallées encaissées du Massif schisteux (Cordier, 2004 ; Harmand, 2004, 2007). La préservation du cône-terrasse de Diefflen peut également s'expliquer par sa position en retrait par rapport à un organisme fluvial puissant tel que celui de la Sarre.

\section{BIBLIOGRAPHIE}

BEINER M., HARMAND D., OCCHIETTI S., CORDIER S. (2009). - Les minéraux lourds des alluvions quaternaires du bassin de la Moselle : nouvelles données. Quaternaire, 20, (4), 63-79.

CORDIER S (2004). - Les niveaux alluviaux quaternaires de la Meurthe et de la Moselle entre Baccarat et Coblence : étude morphosédimentaire et chronostratigraphique, incidences climatiques et tectoniques. Thèse de Géographie physique. Université de Paris XII. Volume texte : 287 p, Volume des illustrations : 116 fig., 25 tab., 10 pl. photos.

CORDIER S., HARMAND D., FRECHEN M., BEINER M., (2006). - New evidences on the Moselle terrace stratigraphy between the Meurthe confluence (Paris basin) and Koblenz (Rhenish Massif), Z. Geomorph. N. F., 50, 3, pp. 281-304.

FISCHER F. (1957). - Beiträge zur Morphologie des Flußsystems der Saar. - Ann. Univ. Sarav., Saarbrücken, 5, pp. 104-192.

HARMAND D. (2004). - Genèse et évolution du réseau hydrographique (creusement des vallées et captures) dans les régions de moyennes latitudes : exemple de l'Est du bassin de Paris. Mémoire d'habilitation à diriger des recherches. Université de Nancy 2, 272 p, 49 fig., 10 tab.

HARMAND D. (2007). - Révision du système des terrasses alluviales de la Sarre entre Sarrebourg (France, Lorraine) et la confluence avec la Moselle à Konz (Allemagne, Rhénanie-Palatinat). Revue Géographie de l'Est, ce volume. 
KONZAN H.-P., KLINHAMMER B., MÜLLER E. (1977). - Erläuterungen zur Geologischen Karte des Saarlandes 1:25 000, Blatt nr. 6606 Saarlouis, 48 S. Saarbrücken. Saarbrücken (Arbeiten aus dem Geographischen Institut der Universität des Saarlandes).

LIEDTKE, Herbert (1998). - Carte géomorphologique à 1/300 000 de la région Saar-Lor-Lux. Geschichtlicher Atlas für das Land an der Saar. H. Quasten und H.-W. Herrmann Ed. Saarbrücken.

NEGENDANK J. (1978). - Zur känozoischen Geschichte von Eifel und Hunsrück. Sedimentpetrographische Untersuchungen im Moselbereich. Forschungen zur deutschen Landeskunde, Band 211, 90 S.

ZÖLLER L. (1984). - Das Quartär-Profil von Dillingen (Saarland). Jber. Mitt. Oberrhein. Geol. Ver., N. F. 66, pp. 351-355.

ZÖLLER L. (1985). - Geomorphologische und quartärgeologische Untersuchungen im HunsrückSaar-Nahe-Raum. Forchungen zur deutschen Landeskunde. 25, 240 S.

\section{RÉSUMÉS}

La Prims, cours d'eau affluent de la Sarre, a édifié au cours du Pléistocène de puissantes formations alluviales notamment à Diefflen, à proximité de Saarlouis (Saarland, Allemagne). Ces dépôts fluviatiles d'environ une dizaine de mètres d'épaisseur, situés à $+60 \mathrm{~m}$ d'altitude relative par rapport à la Prims, sont remarquablement préservés sous trois mètres de loess. Leur étude sédimentologique a mis en évidence le caractère proximal des apports, provenant du socle dévonien de l'Hunsrück ainsi que de sa couverture sédimentaire permienne et triasique (Buntsandstein). L'hétérométrie des sédiments, leur structure, leur faciès, conduisent à attribuer ces formations à des cours d'eau à forte énergie mais épisodiques, dans un contexte paléoenvironnemental périglaciaire, certainement antérieur au Saalien.

The Prims River, a tributary of the Sarre River, built up during the Pleistocene important alluvial formations of about ten meters of thickness near Diefflen (Saarland, Deutschland). These fluvial deposits, situated at $+60 \mathrm{~m}$ above the actual bed of the Prims River, are covered by three meters of loess and still very weil preserved. Sediment analysis showed that they originated from the Devonian Hunsrück massif, as weil as from the Permian basin and its overlying sediments of Triassic age (Bunlsandstein). Granulometry of these sediments (cobbles, gravels, sand and silt), their internai structure and their facies witness that they were formed under highly energetic hydrodynamic conditions, as they were typical during the periglacial climale before the Saalian.

Die Prims, ein Nebenfluss der Saar, hat wahrend des Pleistozans imposante und gut erhaltene alluviale Sedimentformationen aufgeschüttet, die in der Nahe Diefflen (Saarland, Deutschland) eine Machtigkeit von etwa zehn Metern erreichen kiinnen. Sedimentanalysen zeigen, dass sie sowohl aus dem herzynischen Hunsrückgebirge stammen, ais auch von den darüberliegenden Sedimentgesteinen des Perms und der Trias. Die KorngröBe der Sedimente (Kies, Schotter, Sand und Silt) und ihre interne Struktur weisen auf eine Bildung unter ernergiereichen hydrodynamischen Bedingungen hin, die mit Sicherheit einem periglazialen Klima vor der Saalischen Vereisung entsprechen. 


\section{AUTEURS}

\section{PIERRE SERRAT}

EA-1135 CERPA Centre d'études et de recherches sur les paysages - Université Nancy $2-23$, boulevard Albert $1^{\mathrm{er}}-54015$ NANCY cerpa@univ-nancy2.fr

\section{DOMINIQUE HARMAND}

EA-1135 CERPA Centre d'études et de recherches sur les paysages - Université Nancy 2 - 23, boulevard Albert $1^{\mathrm{er}}-54015$ NANCY cerpa@univ-nancy2.fr 